\title{
On initialization of milling paths for 5-axis flank CNC machining of free-form surfaces with general milling tools
}

\author{
Pengbo Bo ${ }^{\mathrm{a}}$, Michael Bartoñ $\check{\mathrm{b}}^{*, \mathrm{~b}}$ \\ ${ }^{a}$ School of Computer Science and Technology, Harbin Institute of Technology, West Wenhua Str. 2, 264209 Weihai, China \\ ${ }^{b}$ BCAM - Basque Center for Applied Mathematics, Alameda de Mazarredo 14, 48009 Bilbao, Basque Country, Spain
}

\begin{abstract}
We propose a path-planning algorithm for 5-axis flank CNC machining with general tools of varying curvature. Our approach generalizes the initialization strategy introduced for conical tools Bo et al. [2017] to arbitrary milling tools. Given a free-form (NURBS) surface and a rotational milling tool, we look for its motion in $3 \mathrm{D}$ to approximate the input reference surface within a given tolerance. We show that for a general shape of the milling tool, there exist locally and generically four 3D directions in which the pointsurface distance follows the shape of the tool up to second order. These directions form a 3D multi-valued vector field and its integration gives rise to a set of integral curves. Among these integral curves, we seek straight line segments that correspond to good initial positions of the axes of the milling tool. We validate our method against synthetic examples with known exact solutions and, on industrial datasets, we detect approximate solutions that meet fine machining tolerances. We also demonstrate applicability of our method for efficient flank milling of convex regions that is not possible using traditional conical tools.
\end{abstract}

Key words: 5-axis CNC machining, flank milling, free-form surface, tangential movability, general milling tool

\section{Introduction \& Motivation}

Computer numerically controlled (CNC) machining is the leading subtractive technology that is used for manufacturing of objects of various shapes and scales, spanning from micro-milling of coins and medals, over moderately-sized blades of turbines, up to huge molds and support structures in free-form architecture [Pottmann et al., 2008]. This brings challenges to geometric modeling and computer aided design as the milling tool needs to be navigated in 3D space to follow very precisely the shape of a designed free-form (NURBS) geometry.

CNC milling techniques can be classified in at least three ways: (i) By the degrees of freedom of the milling machine: 3-axis and 5-axis milling are the most frequently used technologies. (ii) By the shape of the milling tool: flat-end Li and Jerard [1994]; Fan and Ball [2014]; Zhao et al. [2018], ball-end Ikua et al. [2001], bull-nose Campa et al. [2007], and general Engin and Altintas [2001]; Machchhar et al. [2017] cutting tools. (iii) By the nominal dimension of contact between the milling tool and the reference surface; the simplest case is zero-dimensional contact, also known as point milling, where the tool and the surface touch at a single point. In our research, we consider a type of 5-axis milling that considers positioning a general rotational tool in 3D to have one-dimensional contact with the surface, known as flank milling.

Single-point contact milling guarantees good approximation only in the very neighborhood of the contact point. Therefore, extensive research is devoted to increase this neighborhood. For example, in 5 -axis flat-end milling with cylindrical tools, one can use the extra degrees of freedom remaining after posing the contact

\footnotetext{
${ }^{*}$ Corresponding author

Email addresses: pbbo@hit.edu.cn (Pengbo Bo), mbarton@bcamath.org (Michael Bartoň)
} 
(a)

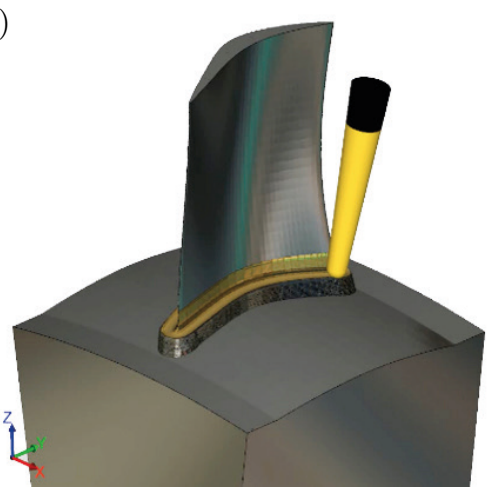

(b)

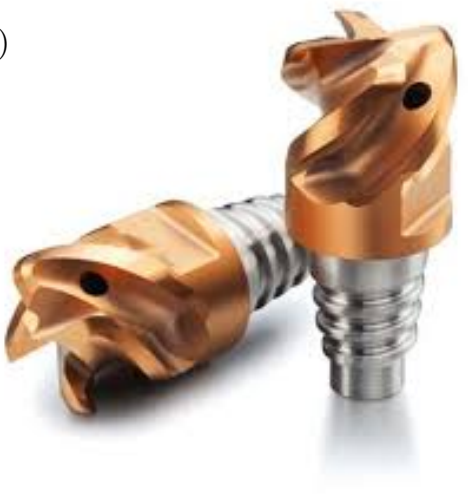

Figure 1: (a) A flank milling simulation by ModuleWorks using conical milling tool. (b) A general, curvature-varying milling tool by Sandvik. Figures courtesy of ModuleWorks GmbH and Sandvik Coromant, respectively.

circle of the cylinder to a single-point contact. Fixing the contact point, two degrees of freedom (known as tilting and rotating angles) can be optimized to achieve a higher order of contact. Consequently, such optimized positioning reduces the error between the designed surface and the milled approximation.

An osculating circle possesses second order contact with the surface. In the context of flat end milling, this approach is known as curvature matching and the position of the tool is sought after such that the contact circle of the tool matches the curvature of the surface in a certain tangent direction. To increase the approximaition quality, one may require higher (third) order contact by considering cases where the contact circle becomes hyperosculating Wang et al. [1993a,b], or to require double-tangential contact Kim et al. [2015]. Even though the approximation quality is increased in all the above cases, conceptually the contact circle and the reference geometry share only finitely many contact points: infinitesimally close three - osculating, four - hyperosculating, or two pairs - double-tangential circles. That is, the dimension of the set of the contact points is zero.

To achieve considerably better approximation quality, one-dimensional contact between the designed and milled surfaces is exploited. The corresponding CNC methodology is flank (aka peripheral) milling Harik et al. [2013]; Senatore et al. [2012a]; Li et al. [2006, 2008]; Zhu et al. [2010]; Gong and Wang [2009]; Zhu and $\mathrm{Lu}$ [2015]; Calleja et al. [2018] and the one-dimensional contact, a 3D curve, is known as characteristic (or grazing curve). Due to the contact at many (theoretically infinitely many) points, flank milling is more efficient in material removal and is by definition scallop-free along each milling path when compared to the single-point milling. However, a general NURBS surface is not an envelope of some surface of revolution and therefore one only seeks patches of the input NURBS surface that can be sufficiently well approximated by envelopes Bo et al. [2016].

Traditionally, the tools for flank milling are mainly cones (cylinder being a special type of a cone with constantly zero slope), i.e., tools whose meridians are planar curves of zero curvature. However, the freeform nature of the reference geometry may be approximated better by a generally curved milling tool. This brings two main challenges in geometric modeling: (i) find an optimal shape of a tool (or a set of tools) that offer the best approximation to a given free-form geometry. (ii) Given a free-form surface and a shape of a general, curvature-varying milling tool, see Fig. 1(b), find its milling paths such that the corresponding envelopes approximate the input surface within fine machining tolerances. Our work tackles the second problem and seeks milling paths of arbitrarily curved milling tools.

\section{Related work \& Contributions}

5-axis flank CNC milling offers many challenging problems in applied geometry and therefore has attracted a lot of research attention, see e.g. Elber [1994]; Senatore et al. [2012b, 2008b]; Redonnet et al. 
[1998]; Pechard et al. [2009]; Warkentin et al. [2000], the survey paper Harik et al. [2013], and the references cited therein. Geometrically, one may conceptualize the problem as follows: given an input free-form surface $\Phi$ and a surface of revolution $\Psi$ with varying curvature (milling tool), approximate $\Phi$ by $3 \mathrm{D}$ rigid body motions of $\Psi$ such that the error (measured e.g. as a one-sided or Hausdorff distance) is below a given numerical threshold $\varepsilon$.

In this paper we use a geometric abstraction of flank milling that sets aside some issues of physical interaction between the tool and surface. In practice, the milling tool is not actually a surface of revolution, but has teeth, see Fig. 1(b), that deal with material removal as the tool spins around its axis. The physical shape of the tool (e.g., the number of teeth) affects cutting force Sun et al. [2013]; Larue and Altintas [2005]; Zhang et al. [2016]; Xu et al. [2017] and vibration Sonthipermpoon et al. [2010]; Lu et al. [2014], which can affect the quality of flank milling. A post-process may need to regulate the speed as the tool follows our tool path. Likewise, we do not consider global collision detection, though one could do so with a bounding volume hierarchy Chang et al. [2010].

Another physical-oriented issue in high-performance manufacturing relates to velocity and acceleration control, see e.g. Beudaert et al. [2012]; De Lacalle et al. [2006]; Sorby et al. [2000], as well as the distribution of the feedrates in the tangential vs. in-milling-axis directions Beudaert et al. [2012]. A trade-off between the geometric accuracy and smoothness of the motion is discussed in Pechard et al. [2009].

Considering mainly the geometric aspects between the designed and milled surface, a lot of research focuses on approximating the input surface by ruled surfaces Flöry and Pottmann [2010]; Li et al. [2006, 2008]; Elber and Fish [1997]; Sprott and Ravani [2008]; Chen and Pottmann [1999]; Gong et al. [2005]; Senatore et al. [2008a]; Chu et al. [2008]; Wang and Elber [2014]. Using divide-and-conquer concept, the input surface is subdivided into smaller segments and each segment is approximated by a bilinear patch to control the approximation quality Elber and Fish [1997]. However, such an approach typically requires a large number of patches to meet fine approximation thresholds.

The fact that the two surfaces stay in a tangential contact is a very strong restriction. However, it offers a very valuable information in the search for good initial positions of the milling axes: a linear segment 1 in $3 \mathrm{D}$ can be the milling axis only if the distance between a point on $\mathbf{l}$ and $\Phi$ changes accordingly to the profile of the milling tool $\Psi$. We exploit this fact and generalize the initialization strategy introduced in Bo et al. [2017] from linear meridians to general ones.

Our approach computes a vector field that consists of directional vectors in which the point-surface distance changes according to the shape of the meridian of the milling tool. We show that in a generic case, there exist four admissible directions in $\mathbb{R}^{3}$ in which the milling tool and the surface match up to second order. We further adopt the methodology introduced in Bo et al. [2017] and seek this multi-valued vector field to compute integral curves in $\mathbb{R}^{3}$ that approximate the axis of the milling tool. Our another contribution that goes beyond Bo et al. [2017] is a search space reduction strategy that quickly eliminates parts of $\mathbb{R}^{3}$ that cannot contribute by good axis positions.

The rest of the paper is organized as follows. We introduce envelopes of surfaces of revolution with curved meridians in Section 3. The reduction of the search space and the fitting algorithm is introduced in Section 4 and the results are shown in Section 5. Finally, the paper is concluded and future research directions are discussed in Section 6.

\section{Envelopes of surfaces of revolution}

Our goal is to approximate a general free-form surface $\Phi$ by an envelope of a general milling tool (surface of revolution) $\Psi$. We consider general, curvature-varying milling tools that are introduced in 3.1. The tangential contact between two surfaces is discussed in 3.2 and the computation of directions in which the distance from $\Phi$ changes according to the shape of $\Psi$ is described in 3.3.

\subsection{Meridian vs. radial function}

A milling tool with a rotational axis $\mathbf{l}$ is a surface of revolution $\Psi$ that can be represented in two ways: using a radial function or a meridian curve. The radial function $r: \mathbf{l} \rightarrow \mathbb{R}^{+}$gives the radius $r(s)$ of a sphere 


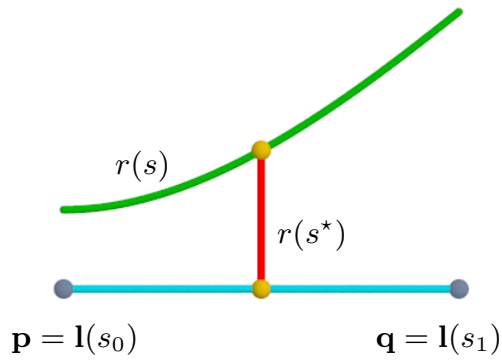

(a)

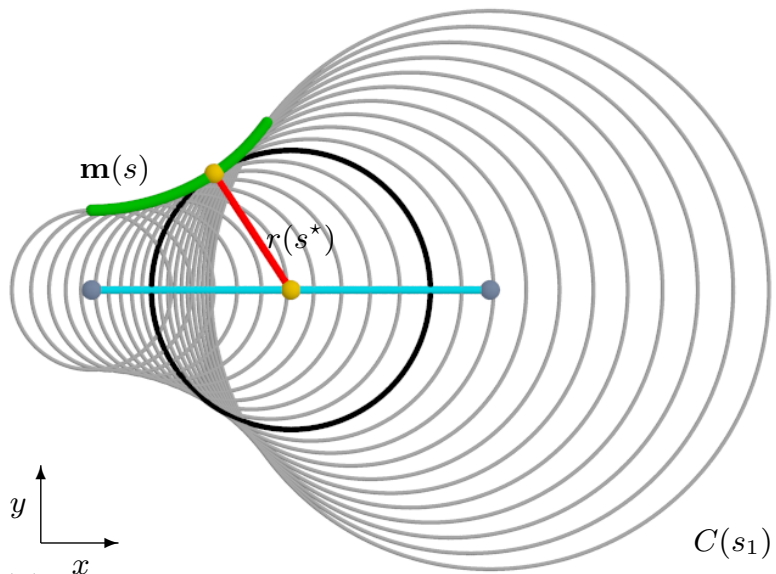

(b)

Figure 2: Radial function $\rightarrow$ meridian. (a) Legitimate radial function $r(s)$ and its value (red) at the midpoint of a line $\mathbf{l}=\mathbf{p q}$. (b) The half-meridian $\mathbf{m}(s)=[x(s), y(s)]$ is an envelope of a one-parameter family of circles centered along $\mathbf{l}, r(s)$ being the radii. Given $r(s), \mathbf{m}$ is parametrized via Eq. (3).

centered at $s \in \mathbf{l}$ and the surface $\Psi$ is the envelope of this one-parameter family of spheres Pottmann and Peternell [2000]. The meridian curve (or meridian, for short) is the intersection of $\Psi$ with an arbitrary plane containing $\mathbf{l}$; it has two symmetric branches.

An example of monotonically increasing radial function is shown in Fig. 2. Alternatively, one may have the half-meridian as an input and needs to compute the radial function from it by parsing the axis and computing the closest points (footpoints) on $\mathbf{m}$ as shown in the following Example.

Example 1. A toroidal milling tool. A half-meridian $\mathbf{m}$ is a circle that does not intersect $\mathbf{1}$, (due to accessibility we consider only the half-circle farther from 1), see Fig. 3. Let $A$ and B be the toroidal raidii, then a simple computation gives the radial function

$$
r(s)=\sqrt{A^{2}+s^{2}}+B
$$

as the parameter $s$ traverses $\mathbf{l}$ ( $s=0$ is the point on $\mathbf{l}$ that is closest to the center of the circle).

For toroidal milling tools, the conversion of the meridian to the radial function is rather straightforward, however, for a general meridian such a conversion requires computation of footpoints which is less convenient and therefore, from now on, we assume that the radial function $r(s)$ is our input to define $\Psi$.

Assume that the axis $\mathbf{l}$ coincides with the $x$-axis and is parameterized by $\operatorname{arc-length} \mathbf{l}(s), s \in\left[s_{0}, s_{1}\right]$, see Fig. 2(a). Given a radial function $r(s)$, the computation of a half-meridian $\mathbf{m}(s)=[x(s), y(s)]^{\mathrm{T}}$ follows the fact that the meridian points are the intersections of two infinitesimaly close circles

$$
\begin{aligned}
(x(s)-s)^{2}+y^{2}(s) & =r^{2}(s), \\
(x(s)-s-\Delta s)^{2}+y^{2}(s) & =r^{2}(s+\Delta s),
\end{aligned}
$$

with the incremental step $\Delta s$. Solving Eq. (2) as $\Delta s \rightarrow 0$ gives the meridian parametrization

$$
\mathbf{m}(s)=\left[\begin{array}{c}
s-r(s) r^{\prime}(s) \\
r(s) \sqrt{1-r^{\prime 2}(s)}
\end{array}\right] .
$$

Observe that if $\left|r^{\prime}(s)\right|>1$, the $y$-coordinate is complex and therefore the envelope is not a real curve anymore. The reason for that is that the radius increases (decreases) too quickly when compared to the motion of the parameter $s$ and consequently the sequence of circles (and spheres) is nested. If $r^{\prime}(s) \equiv 1$, 


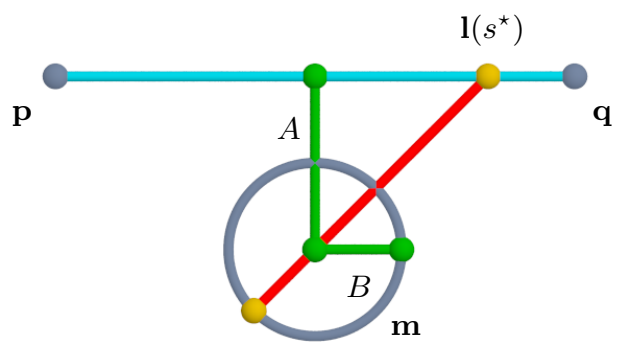

(a)

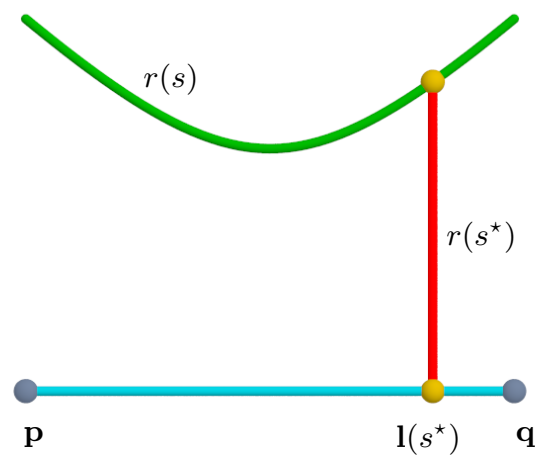

(b)

Figure 3: A toroidal milling tool. (a) The half-meridian $\mathbf{m}$ of a toroidal milling tool with radii $A$ and $B$. The axis $\mathbf{l}=\mathbf{p q}$ is parametrized and the distance to a footpoint on $\mathbf{m}$ defines the radial distance $r\left(s^{\star}\right)$ (red). Due to accessibility, only the footpoints on the half-circle farther from 1 need to be considered. (b) The radial distance function $r(s)$ (green) of the toroidal milling tool, see Eq. (1).

the circles form a parabolic pencil passing through the origin. Such situations shall be excluded from the definition of a meridian. A more detailed discussion on 2D envelopes can be found, e.g., in Farin et al. [2002].

For the simplicity of argument, we further assume that the radius function is non-decreasing (otherwise we can flip the parametrization) because we want to have a one-to-one correspondence between the points on $\mathbf{l}$ and $\mathbf{m}$. Altogether, we require

$$
0 \leq r^{\prime}(s)<1
$$

and radial functions satisfying Eq. (4) will be called legitimate.

Remark 1. If $r(s)$ is not injective $\left(r^{\prime}(s)<0\right)$, then for the affected radius $r^{\star}$, there exist two mutually different parameter values $s_{1}^{\star}, s_{2}^{\star}$ such that $r^{\star}=r\left(s_{1}^{\star}\right)=r\left(s_{2}^{\star}\right)$. This means that for a given point $\mathbf{p} \in \mathbf{l}$ such that its distance is $r^{\star}$, there exist (at least) two points on the meridian and therefore the position of $\Psi$ w.r.t. $\Phi$ is not unique. Such a case requires a special treatment, but as shown later in Section 5 , we can handle also this type of meridians.

\subsection{Tangential contact between two surfaces}

We aim to pose the milling tool $\Psi$ such that it touches $\Phi$ tangentially, that is, in an ideal case $\Psi$ and $\Phi$ have a $3 \mathrm{D}$ curve in common. The wish of a shared curve can be locally formulated using higher order contact between the tool and the surface. To achieve this goal, we approximate the distance between a point and $\Phi$, and using this approximation, we can further compute $3 \mathrm{D}$ vectors that correspond to directions of the milling axis such that the tool $\Psi$ has the second order contact with $\Phi$. First we define the distance function.

Let $d(\mathbf{p}, \Phi)$ be the distance function that assigns to a point $\mathbf{p} \in \mathbb{R}^{3}$ its (signed) distance from an oriented surface $\Phi$, i.e.,

$$
\mathbb{R}^{3} \stackrel{d}{\longrightarrow} \mathbb{R}
$$

and let $\Psi$ be a surface of revolution with a rotational axis 1 . If $\Phi$ and $\Psi$ have a tangential contact, then, for a point $\mathbf{p}$ moving along $\mathbf{l}$, its distance to $\Phi$ has to follow the radial function $r(s)$ that governs the shape of $\Psi$. Let

$$
d(\mathbf{p}, \Phi)=r\left(s^{\star}\right) \quad \text { for some } \quad s^{\star} \in\left[s_{0}, s_{1}\right],
$$

then we seek a unit vector(s) $\mathbf{v}$ that satisfy

$$
\begin{array}{ccc}
\nabla_{\mathbf{v}} d & = & r^{\prime}(s) \\
\nabla_{\mathbf{v}}\left(\nabla_{\mathbf{v}} d\right)=\nabla_{\mathbf{v}}^{2} d & = & r^{\prime \prime}(s)
\end{array}
$$




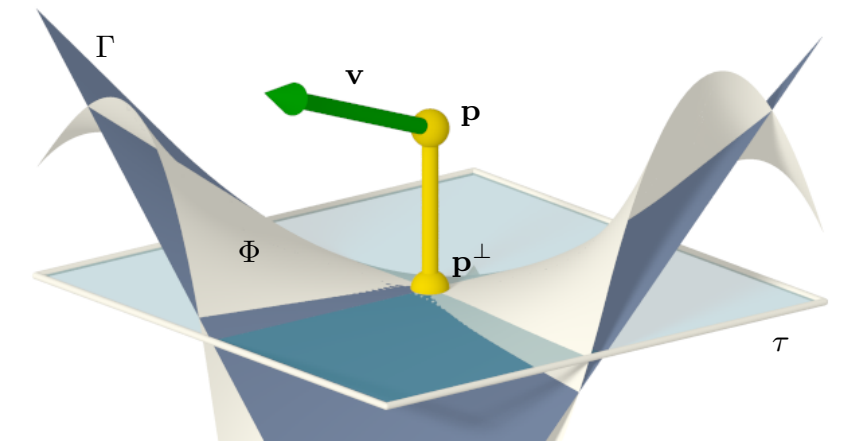

Figure 4: Second order surface approximation. Given a point $\mathbf{p}$ outside $\Phi$, its distance to $\Phi$ is approximated using the osculating paraboloid $\Gamma$ of $\Phi$ at the footpoint $\mathbf{p}^{\perp}$. The distance $\left|\mathbf{p} \mathbf{p}^{\perp}\right|$ has to be equal to the milling radius $r\left(s^{\star}\right), s^{\star} \in\left[s_{0}, s_{1}\right]\left(\right.$ zero-order $^{\circ}$ contact), and we look for 3D vector(s) (green) in which the point-surface distance changes according to Eq. (7), resulting in second order contact between $\Phi$ and $\Psi$.

which expresses our wish that the distance function matches the radial function up to second order, see Fig. 4. Looking for unit $\mathbf{v}$, Eqns. (6) and (7) define a second order initial value problem and its solutions are integral curves in 3D.

To compute an accurate approximation of $\Phi$ by $\Psi$, we use second order approximation of the distance function $d$. Let $\mathbf{p} \in \mathbf{l}, \mathbf{p}^{\perp}$ be its closest point on $\Phi$, and $\left\{\mathbf{d}_{1}, \mathbf{d}_{2}, \mathbf{n}\right\}$ be the principal frame at $\mathbf{p}^{\perp}$. Then, writing $\mathbf{p}$ in the coordinate system associated to this frame as $\mathbf{p}=[0,0, h]^{\mathrm{T}}$, the second order approximation of the distance function reads

$$
\widetilde{d}(\mathbf{x}, \Gamma)=\frac{1}{2\left(h-\rho_{1}\right)} x_{1}^{2}+\frac{1}{2\left(h-\rho_{2}\right)} x_{2}^{2}+x_{3},
$$

where $\Gamma$ is the osculating paraboloid of $\Phi$ at $\mathbf{p}^{\perp}$ and $-\rho_{1,2}$ are the radii of the osculating circles of $\Psi$ in the principal normal planes, see [Bo et al., 2017, Section 3.3] for more details.

\subsection{Admissible directions}

We are given a radial function $r(s), s \in\left[s_{0}, s_{1}\right]$ and we assume that for a given distance $h=\left\|\mathbf{p} \mathbf{p}^{\perp}\right\|$, we can compute $s^{\star}$ such that $r\left(s^{\star}\right)=h$ and also to compute $r^{\prime}\left(s^{\star}\right)$ and $r^{\prime \prime}\left(s^{\star}\right)$. We seek unit directional vector $\mathbf{v}=\left[v_{1}, v_{2}, v_{3}\right]^{\mathrm{T}}$ that solves Eq. (7). Computing the first directional derivative of $\widetilde{d}(\mathbf{x}, \Gamma)$ at $\mathbf{p}$ gives

$$
\nabla_{\mathbf{v}}(\widetilde{d}(\mathbf{p}))=\frac{v_{3}}{\|\mathbf{v}\|}=v_{3}
$$

which, using Eqns. (7), (8), and the fact that $\|\mathbf{v}\|=1$ gives

$$
\begin{array}{ccc}
v_{1}^{2}+v_{2}^{2} & =1-r^{\prime}(s)^{2}, \\
\frac{v_{1}^{2}}{r(s)-\rho_{1}}+\frac{v_{2}^{2}}{r(s)-\rho_{2}} & =r^{\prime \prime}(s),
\end{array}
$$

where $-\rho_{1,2}$ are the principal curvature radii of $\Phi$ at $\mathbf{p}^{\perp}$.

Given the values of $r, r^{\prime}$, and $r^{\prime \prime}$ at some known $s \in\left[s_{0}, s_{1}\right]$, Eq. (10) is a well-constrained $(2 \times 2)$ system with $v_{1}$ and $v_{2}$ as the unknowns. Observe that the normalization constraint is already incorporated there (see Eq. (9) and the first equation of Eq. (10)). Geometrically, we can interpret the problem as an intersection of two implicit quadrics and the unit sphere (normalization constraint) in $\mathbb{R}^{3}$, see Fig. 5. The quadrics are a rotational cone and a cylinder (hyperbolic or elliptic depending geometry of $\Phi$ ).

Remark 2. We seek an intersection of three quadrics in $\mathbb{R}^{3}$ which by Bézout theorem has at most eight real solutions under the assumptions that neither of them have a common component. Due to symmetries, the problem reduces to intersection of a circle with a conic, see Eq. (10), that in general has four intersections symmetric w.r.t. the principal directions $\mathbf{d}_{1}$ and $\mathbf{d}_{2}$. 


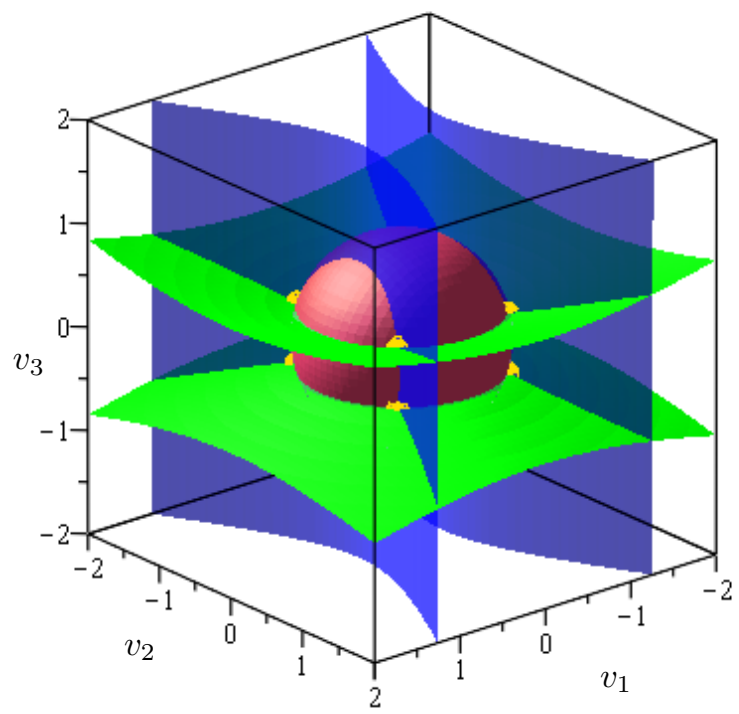

Figure 5: The system Eq. (10) together with the normalization $\|\mathbf{v}\|=1$ (red) results in a cone-sphere-cylinder intersection in the $\left[v_{1}, v_{2}, v_{3}\right]$-space; here the surface geometry results in a hyperbolic cyllinder (blue). Generically, there are eight solutions (yellow) that, due to symmetry, define four directions in which the point-surface distance changes according to the meridian of the milling tool.

Observe that Eq. (10) is linear in $v_{1}^{2}$ and $v_{2}^{2}$ and therefore we obtain

$$
\begin{aligned}
& v_{1}= \pm \sqrt{\frac{-\alpha_{1}}{\beta}}, \\
& v_{2}= \pm \sqrt{\frac{-\alpha_{2}}{\beta}} .
\end{aligned}
$$

where

$$
\begin{aligned}
\alpha_{1} & =\left(r^{2}-1\right)\left(\rho_{1}-r\right)-r^{\prime \prime}\left(r^{2}+\rho_{1} \rho_{2}\right)+r^{\prime \prime} r\left(\rho_{1}+\rho_{2}\right) \\
\alpha_{2} & =\left(r^{\prime 2}-1\right)\left(r-\rho_{2}\right)+r^{\prime \prime}\left(r^{2}+\rho_{1} \rho_{2}\right)-r^{\prime \prime} r\left(\rho_{1}+\rho_{2}\right) \\
\beta & =\rho_{1}-\rho_{2} .
\end{aligned}
$$

Remark 3. If the footpoint $\mathbf{p}^{\perp}$ is umbilical at $\Phi\left(\rho_{1}=\rho_{2}\right)$, then the second equation in Eq. (10) represents also a right circular cone. In such a case, Eq. (10) has infinitely many solutions if $1-r^{\prime 2}=r^{\prime \prime}\left(r-\rho_{1}\right)$ (the two cones coincide), or there is only a trivial solution $\mathbf{v}=[0,0,0]$. Since the second option is more likely to happen, the computation of the integral curve is terminated if $\mathbf{p}^{\perp}$ is umbilical.

\section{Envelope fitting}

To compute highly accurate approximations of $\Phi$, we propose an algorithm that looks for straight lines in 3D that admit tangential motion along $\Phi$ and the distance changes according to the shape of the tool. Our algorithm consists of quick elimination of the space which cannot contribute by good lines, computation of integral curves, movability analysis, line clustering, and finally global optimization.

\subsection{Search space reduction and exploration}

In order to find good positions of the milling tool's axis, we sample the neighborhood of the reference surface $\Phi$. We perform this sampling in two steps: (i) we segment the input surface using variational shape 

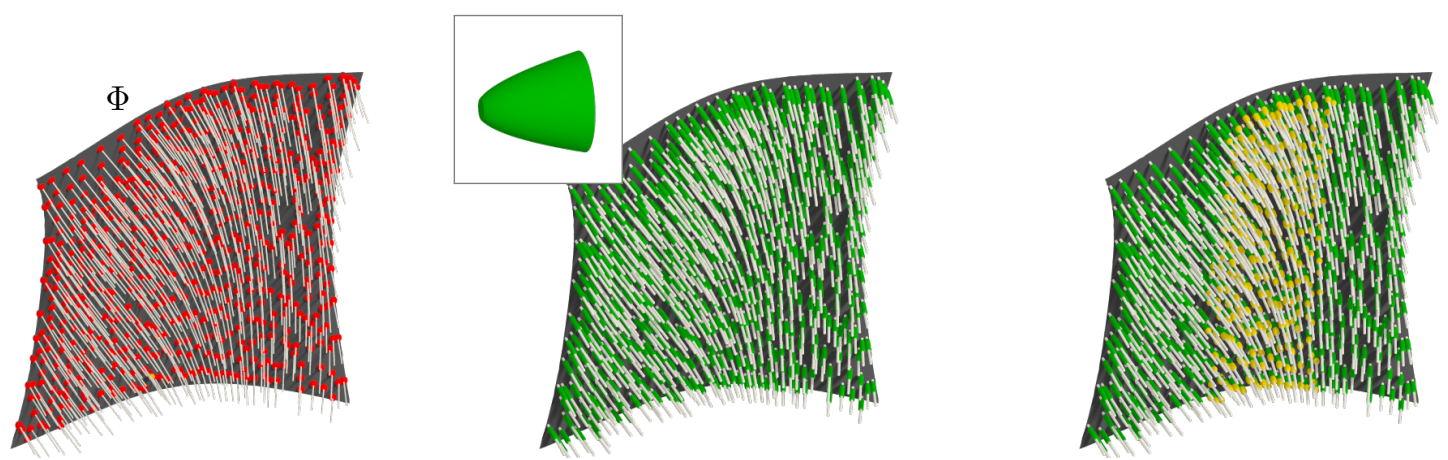

Figure 6: A search space reduction. Left: The normal field (white) of the reference surface $\Phi$ is shown; the seed point sampling is based on the VSA segmentation. Middle: For a given tool (top framed), the radial distance $r(s)$ determines the range $\left[r_{\min }, r_{\max }\right]$ (green) for the possible axial points. Right: this green search space is considerably reduced by requiring real admissible directions (yellow), see Eq. (11).

approximation (VSA) Alliez et al. [2007] and (ii) we sample the points on the surface normals of each segmented polygon.

(i) VSA segmentation is based on a special metric that considers not only the Euclidean distance between points, but also deviation of normal fields. Sampling of the input surface based on VSA is shown in Fig. 6 left; the red points being the barycenters of the proxys (polygons). Such a segemntation corresponds to the best polygonal approximation (of $\Phi$ ) with $k=700$ faces with respect to the $\mathcal{L}^{2,1}$ metric, see Alliez et al. [2007] for more details on VSA.

(ii) On each normal line, we seek points that can contribute as the points of the milling axis. Given a shape of the milling tool (by knowing the radial function $r(s)$ ), one directly knows the distance range $\left[r_{\min }, r_{\max }\right]$ in the normal directions of $\Phi$, see Fig. 6 middle, and the distance range is sampled by $N=30$ seed points.

Observe in Fig. 6 that the sampling range described above admits a huge part of the 3D neighborhood of the reference surface. To quickly eliminate regions in the neighborhood of the surface $\Phi$ that cannot contribute by good seed points, we consider the admissible directions introduced in Eq. (11). If there are no real solutions, the shape of the meridian does not admit higher order approximation of the surface $\Phi$. The admissible directions arise from the circle-vs-conic intersection problem Eq. (10). Therefore it is very easy to check if there are real intersection points or not.

In particular, the radius of the circle is $\sqrt{1-r^{\prime 2}}$ and the main half-axis, e.g., of a hyperbola is $\sqrt{\left(r-\rho_{1}\right) / r^{\prime \prime}}$ (for $r>\rho_{1}, r<\rho_{2}, \rho_{1}>0, r^{\prime \prime}>0$ ). If the hyperbola's main half-axis is larger than the circle radius, there are no real intersection points and consequently no admissible directions. A result of this elimination process is shown in Fig. 6 right.

\subsection{Integral curves}

The analysis introduced in Section 3.3 is used to compute admissible directions in which the distance function between $\Phi$ and $\mathbf{l}$ changes according to the given radial function $r$. The neighborhood of $\Phi$ that cannot contribute by good axis points (complex roots of Eq. (11)) is quickly eliminated and the surface normals are sampled only in those regions where real admissible directions exist, see Fig. 6.

The seed points are guaranteed to have generically four (in the limit two) real directions and integrating this multi-valued vector field of admissible directions gives rise to a family of integral curves, see Fig. 7. Note that these integral curves may be highly curved in some regions. However, we are interested only in those segments that are (almost) straight lines as they corresponds to positions of the milling axis. Therefore, we proceed with the integration of the vector field as follows:

Given an initial point $\mathbf{p}_{i}$ and an initial admissible directional vector $\mathbf{v}_{i}$, we integrate the multi-valued vector field, looking for a consecutive admissible vector $\mathbf{v}_{i+1}$ that points in the almost same direction as $\mathbf{v}_{i}$. This aim is expressed as

$$
\operatorname{angle}\left(\mathbf{v}_{i}, \mathbf{v}_{i+1}\right)<\varepsilon_{\text {linear }},
$$




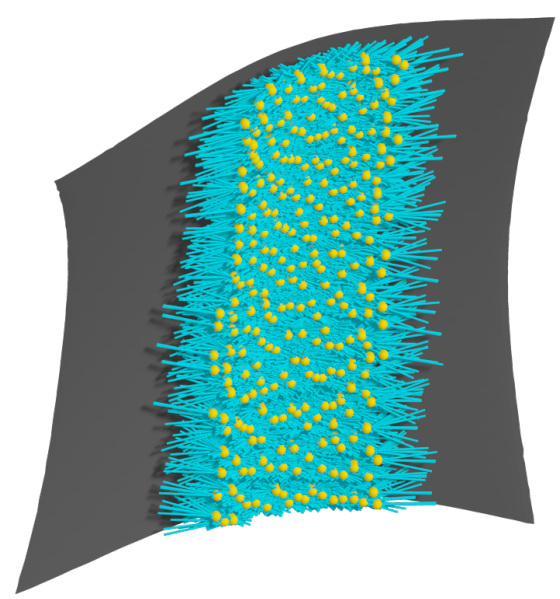

Figure 7: The seed points from admissible range interval (yellow), see also Fig. 6 right, give rise a family of integral curves (blue). These curves are computed by integrating the multi-valud vector field of admissible directions (Eq. (11)).

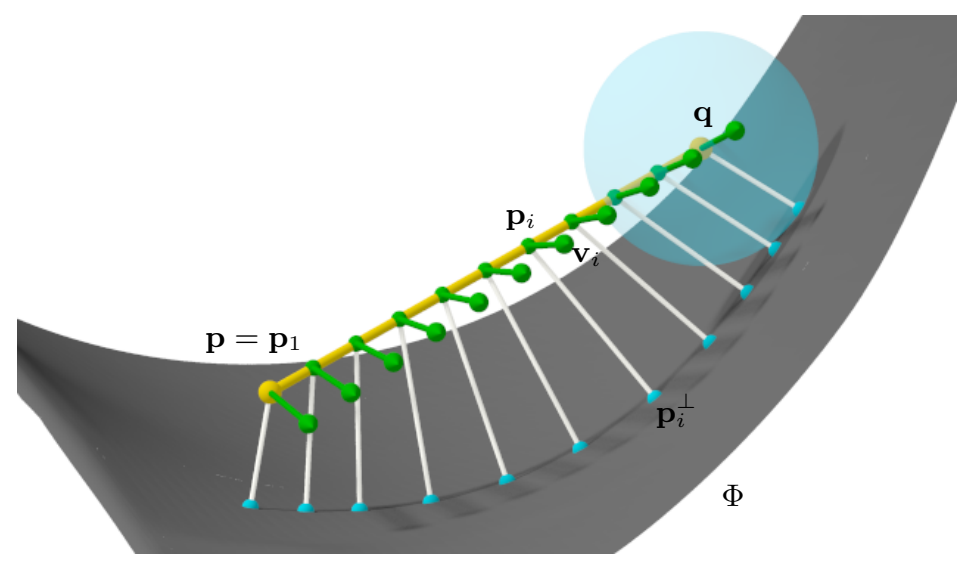

Figure 8: Tangential movability. For flank milling, the milling tool has to move tangentially along $\Phi$. The tool is conceptualized as a one-parameter family of spheres centered along pq (sphere centered at $\mathbf{q}$ is shown in transparent) and an instantaneous motion (green) should move the spheres tangentially along $\Phi$. This objective is interpreted as the orthogonality between the velocity vector $\mathbf{v}_{i}$ at $\mathbf{p}_{i}, \mathbf{p}_{i} \in \mathbf{p q}$, and the line $\mathbf{p}_{i} \mathbf{p}_{i}^{\perp}$. The orthogonality is achieved in the least square sense and the "as orthogonal as possible" vector field is computed from Eq. (17).

where $\varepsilon_{\text {linear }}$ is a parameter that controls the straightness of the integral curve. We set the default value to $\varepsilon_{\text {linear }}=2^{\circ}$ for all the examples in our paper. The integration terminates once the condition in Eq. (13) is violated. The resulting curve is a nearly straight polyline that is approximated by a single linear segment. We use a linear least squares fitting, see e.g. Várady and Martin [2002] for alternative curve fitting algorithms.

\subsection{Tangential movability analysis}

Let $\mathbf{l}:=\mathbf{p q}$ be a finite line in 3D considered as a function of time $t$. Denote $\mathbf{p}(t)$ and $\mathbf{q}(t)$ the trajectories of the endpoints, $t \in[0,1]$. Since 1 remains rigid during the motion, the distance-preserving constraint of its length $d=\|\mathbf{p}(t)-\mathbf{q}(t)\|$ reads as

$$
\|\mathbf{p}-\mathbf{q}\|^{2}=\langle\mathbf{p}-\mathbf{q}, \mathbf{p}-\mathbf{q}\rangle=\text { const. }
$$

where $\langle\cdot, \cdot\rangle$ is the scalar product. Differentiating with respect to $t$ and denoting the velocity vectors by $\mathbf{v}_{\mathbf{p}}=\dot{\mathbf{p}}(t), \mathbf{v}_{\mathbf{q}}=\dot{\mathbf{q}}(t)$, the first order length-preserving constraint of $\mathbf{l}$ becomes

$$
\left\langle\mathbf{v}_{\mathbf{p}}, \mathbf{p}-\mathbf{q}\right\rangle=\left\langle\mathbf{v}_{\mathbf{q}}, \mathbf{p}-\mathbf{q}\right\rangle
$$




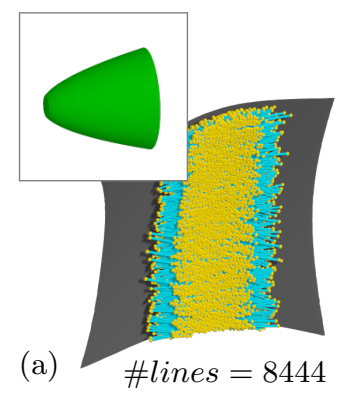

(b)

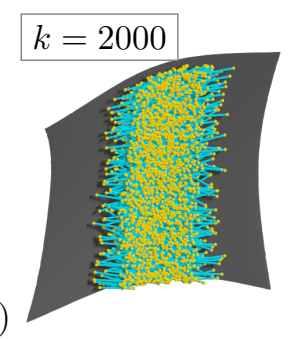

(c)

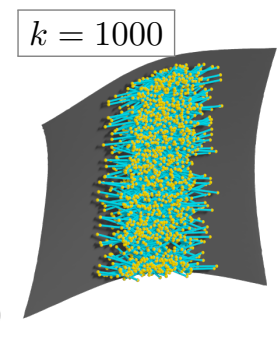

(d)

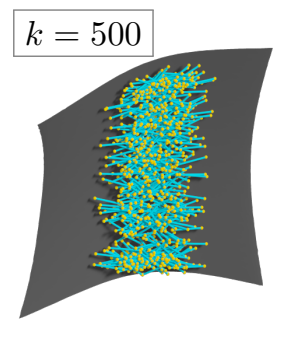

Figure 9: Line clustering. (a) Integrating the multi-valued vector field of admissible directions gives rise a family of integral curves, and those that are almost straight are approximated by a single line. This dense set is clustered using $k$-means clustering method Kanungo et al. [2002]. Results for various $k$ are shown (b-d).

which is known as a projection rule.

We say a line $\mathbf{l}$ is infinitesimally tangentially movable along a surface $\Phi$ if for every point $\mathbf{p}_{i} \in \mathbf{l}$, its closest point $\mathbf{p}_{i}^{\perp}, \mathbf{p}_{i}^{\perp} \in \Phi$, and the instantaneous velocity vector $\mathbf{v}_{i}$ of $\mathbf{p}_{i}$ holds

$$
\left\langle\mathbf{p}_{i}-\mathbf{p}_{i}^{\perp}, \mathbf{v}_{i}\right\rangle=0 .
$$

Note that Eq. (16) holds only if $\Phi$ is infinitesimally an envelope. For a general free-form input, we aim to approximate the best tangential motion. This aim is formulated as a constrained optimization problem

$$
F(\mathbf{x})=\frac{1}{n} \sum_{i=1}^{n}\left\langle\mathbf{v}_{i}, \frac{\mathbf{p}_{i}-\mathbf{p}_{i}^{\perp}}{\left\|\mathbf{p}_{i}-\mathbf{p}_{i}^{\perp}\right\|}\right\rangle^{2}=\mathbf{x} \mathbf{A} \mathbf{x}^{\mathrm{T}} \rightarrow \min ,
$$

with the constraint

$$
\mathbf{v}_{\mathbf{m}} \mathbf{v}_{\mathbf{m}}^{\mathrm{T}}=1
$$

where $\mathbf{x}$ is the unknown instantaneous motion of $\mathbf{l}, n$ is the number of sampled points $\mathbf{p}_{i}$ on $\mathbf{l}, \mathbf{p}_{i}^{\perp}$ are their orthogonal projections onto $\Phi, \mathbf{v}_{i}$ are the velocity vectors associated to $\mathbf{p}_{i}$, and $\mathbf{v}_{\mathbf{m}}$ is the velocity at the midpoint $\mathbf{m}$ of $\mathbf{l}$, see Fig. 8. $\mathbf{x}$ is a five-dimensional vector that describes an instantaneous motion of $\mathbf{p q}$; three parameters are needed to specify the instantaneous velocity at $\mathbf{p}$ and, due to Eq. (15), only two free parameters remain for velocity at $\mathbf{q}$. $\mathbf{A}$ is, in general, a symmetric, positive-definite matrix.

Observe that the objective function $F$ is quadratic and has a trivial minimizer $(\mathbf{x}=[0,0,0,0,0])$. The purpose of the constraint (18) is to eliminate this trivial minimizer that geometrically represents a degenerate, zero-vector field that keeps 1 static. Minimizing Eq.(17) with (18) yields a generalized eigenvalue problem that has a unique non-trivial minimizer $\mathbf{x}^{*}$. If $\Phi$ is an exact envelope, Eq. (16) holds for every point $\mathbf{p}_{i}$ and therefore $F\left(\mathbf{x}^{*}\right)=0$. If $\Phi$ is a general free-form surface that is not an envelope, the minimization (17) seeks the instantaneous motion that is as tangential as possible. The lines with low values of $F$ are said to be tangentially movable along $\Phi$.

\subsection{Line clustering and global optimization}

Once a family of straight lines of the same length (Section 4.2) that admit tangential motion along $\Phi$ (Section 3.2) is obtained, one needs to extract a smaller subset. Typically, this family is very large and we use $k$-means clustering to reduce its size. Several lines' extractions for various $k$ and the milling tool analyzed in Fig. 6 are shown in Fig. 9.

The clustered subsets are sequentially used to form initial motions of the tool. This requires to group and order the lines according to a proximity measure [Bo et al., 2017, Section 4.6]. Finally, the tool paths are globally optimized to minimize the one-sided distance error between the envelope approximation and the reference surface. As this part of the algorithm does not depend whether the shape of the milling tool is conical or not, our approach closely follows [Bo et al., 2017, Section 4.6-7] and we refer the reader to that paper. 

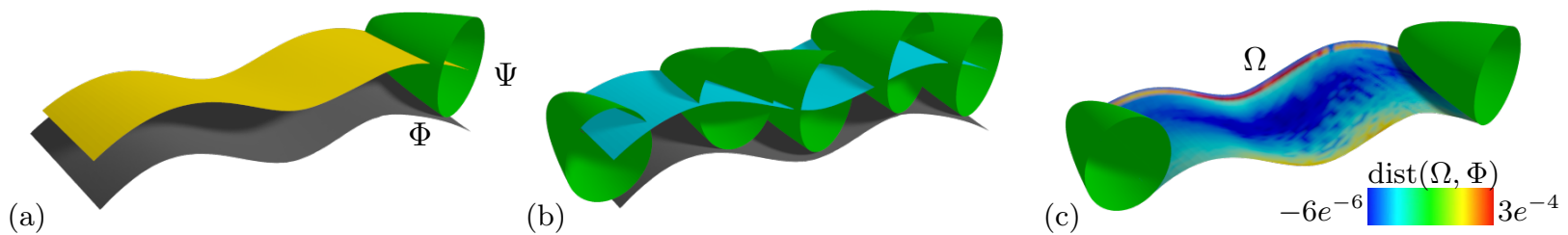

Figure 10: An exact envelope. (a) The reference surface $\Phi$ (dark) is an exact envelope of a motion of a tool $\Psi$ with a general meridian (green). The motion is visualized as a ruled surface (yellow). (b) The exact solution is almost recovered by our algorithm. (c) Our solution, an envelope $\Omega$, is color-coded by the one-sided distance error from the input surface $\Phi$.

(a)

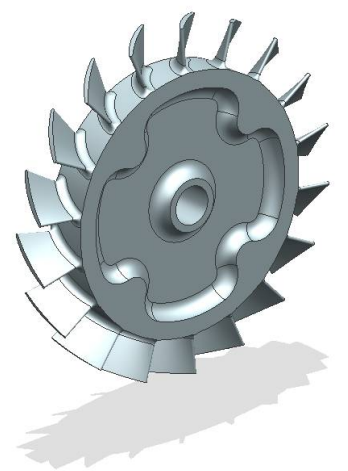

\section{Examples}

We tested our algorithm on benchmark industrial datasets as well as on synthetic examples with known solutions. Fig. 10(a) shows an input surface $\Phi$ that is an exact envelope generated by sweeping a curvaturevarying tool in space. Our solution reproduces the motion up to a very fine tolerance, see Fig. 10(b,c).

We further performed a series of tests on the machining benchmark dataset, the blade of blisk, see Fig. 11. Various types of tool were tested. Fig. 12 shows the results of the three curvature-varying tools, where the radial function $r(s)$ ( $s$ being the arc-length of the tool's axis) is defined analytically as

$$
r(s)=\frac{\sqrt[d]{a+b s}}{c}
$$

with the shape parameters $a, b, c, d \in \mathbb{R}$. Four large envelope patches for each milling tool are shown in Fig. 12. The length of the tool's axis (the $s$ parameter) is set the same for all three tools. While the search for an optimal shape of the milling tool goes beyond the scope of this paper, one can clearly observe very diverse results in terms of approximation quality and surface coverage depending on the tool's shape.

The error $\varepsilon$ between our envelope approximation $\Omega$ and the reference surface $\Phi$ is defined as

$$
\varepsilon=\min _{i, j}\left(\operatorname{dist}\left(\mathbf{r}_{i j}, \Phi\right)-d_{j}\right), \quad i=1, \ldots, m, j=1, \ldots, n
$$

measured over a discrete set of samples $\mathbf{r}_{i j}$ of the ruled surface $R ; d_{j}$ are the distance values obtained from the radial function. We uniformly sample $m=100$ (time) and $n=30$ (ruling direction) in all our experiments.

The errors are measured relatively to a normalized (unit diagonal) bounding box of the blade. Our algorithm returns a set of envelope patches that meet fine machining tolerances that are tenths of milimeters for the blisk of size of tens of centimeters.

In our approach, collision detection is tested as a post-process and is not a part of the optimization. That is, the tool paths that meet the desired accuracy are a-posteriori checked. For example, the last position 

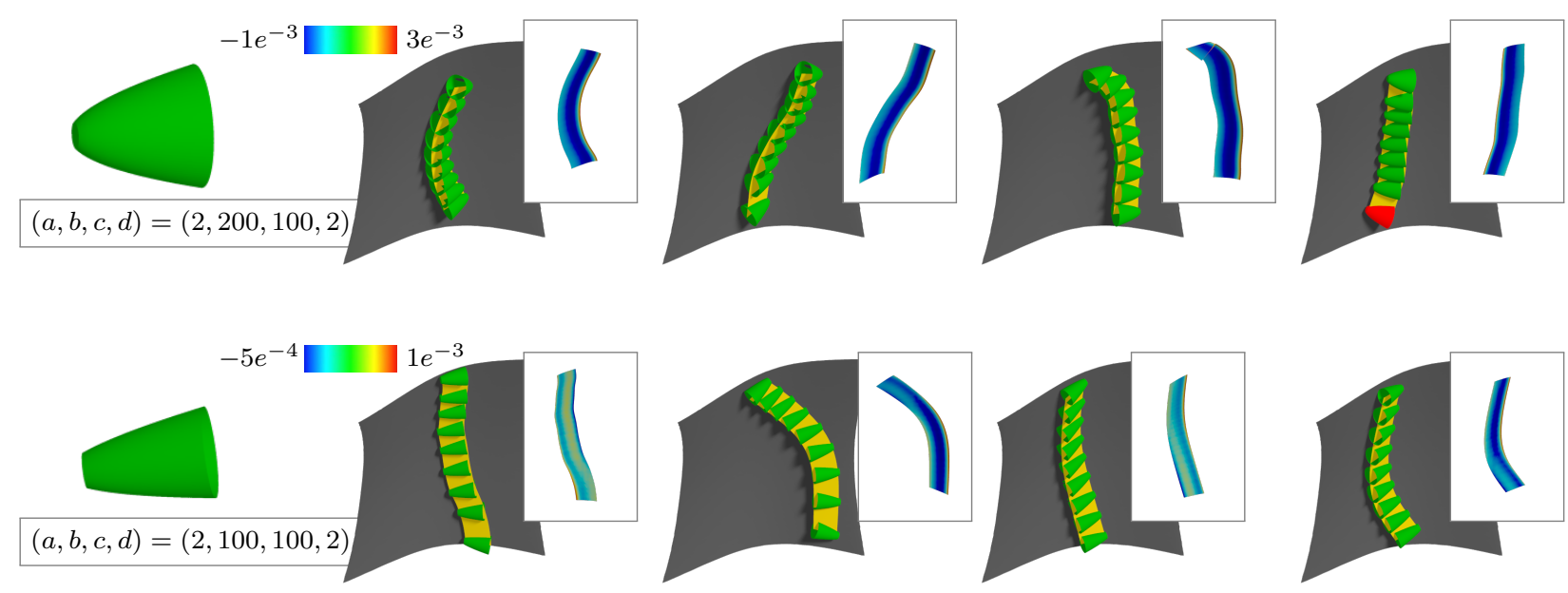

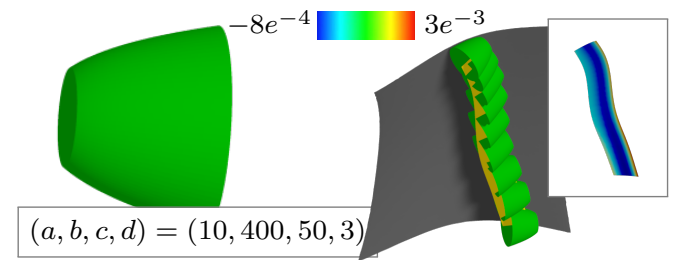

(a)

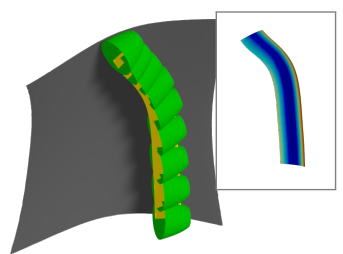

(c)

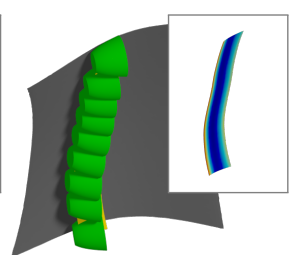

(d)

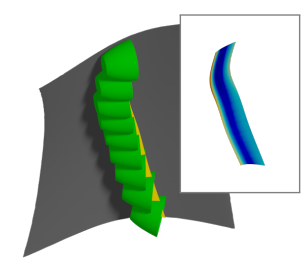

(e)

Figure 12: Shape (and size) matters. (a) Three curvature-varying custom-shaped milling tools are shown together with the vector of shape parameters $(a, b, c, d)$, see Eq. (19). For each tool, four large sweep motions are shown (b-e). The framed pictures are the envelope approximations, color-coded by the one-sided distance error Eq. (20). Observe various error thresholds for each of the three error-bars (applied to each particular tool), indicating that the sweeps of the middle tool possess the best approximation quality. The red tool (top right) corresponds to a scenario when the tool handler collides with the material pedestal.
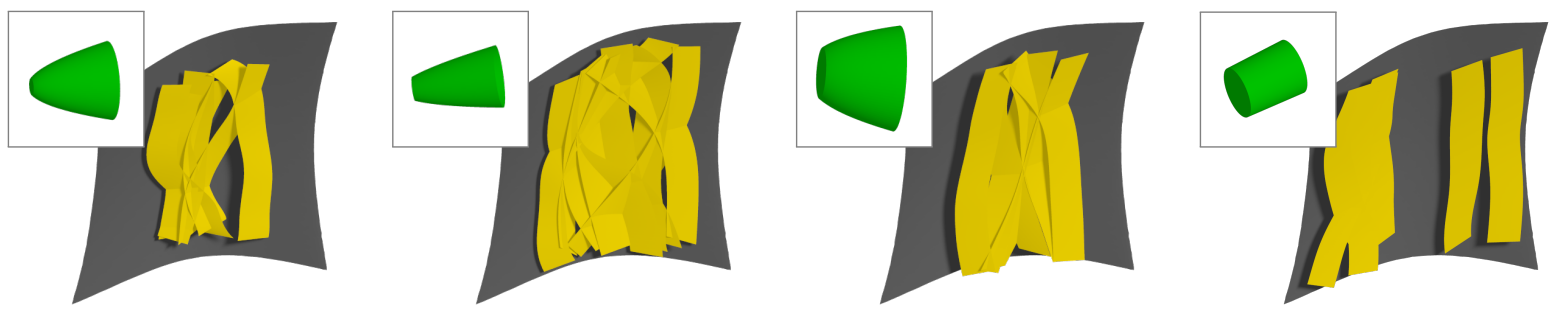

Figure 13: The coverage. The three tools from Fig. 12 are tested in terms of coverage. The milling paths of the tool's axis (yellow) are shown. All envelope patches meet the over- and under-cut machining tolerance, i.e., $|\varepsilon|<1 e^{-3}$ in Eq. (20). The tool at very right is conical: opening angle $\gamma, \sin (\gamma)=0.03$, length of the aixs $d=0.1$, radial distance at the midpoint $r(\mathbf{m})=0.04$. The tool paths were computed using Bo et al. [2017]. The middle (concave) part of the reference surface cannot be well approximated by large sweeps of conical tools.

of the first tool is eliminated, see Fig. 12 top right, because the handler of the tool penetrates the material block at the bottom, see also Fig. 11 (b). But we emphasize that the collision detection was not the main objective of this research and the very global collision detection test whether the tool handler penetrates the neighboring blade has not been considered in this work. For collision detection literature, we refer the reader e.g. to Redon et al. [2005] and the references therein.

We further tested the tools in terms of surface coverage, see Fig. 13. The coverage is visualized using the tool paths (yellow ruled surfaces). Only large patches are shown. The upper bound on overcut error was to $1 e^{-3}$. In this comparison the tool with the shape parameters $(a, b, c, d)=(2,100,100,2)$ (Fig. 13 middle) performs the most favorably, covering almost $85 \%$ of the reference blade. We compared our approach with Bo 

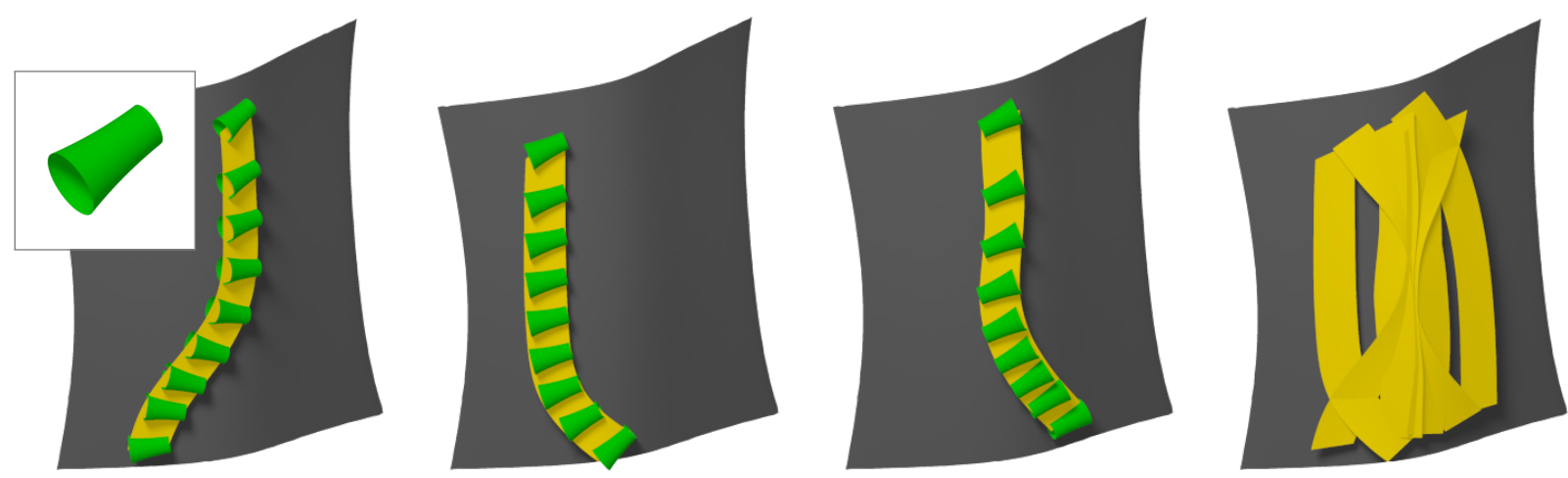

Figure 14: A hyperbolic tool. The convex side of the blade requires tools with negative Gaussian curvature. A hyperbolic tool with the quadratic radial function $r(s)=(0.01+s)^{2}+0.02$ (top framed) admits better approximation than convex tools that were used for the concave side of the blade, c.f. Fig. 12. Three largest tool paths are shown together with a surface coverage by six largest patches (right).

et al. [2017] using a semi-cylindrical (conical with a tiny slope) tool. The results clearly show the limitation of the conical tool in the middle part of the reference surface that is concave and where highly-accurate approximation using conical tools is not possible.

We also experimented with hyperbolic milling tools as shown in Fig. 14. Such tools are well-suited for convex regions of the reference surface. The computation of the admissible directions (see Section 3.3), however, requires a special treatment as the radial function $r$ is not injective. Therefore one needs to select the correct derivative $r^{\prime}$ to the value of $r$ as there are (in our example) two possible values. In our implementation, when tracing the integral curves, we store also the value of $r^{\prime}$ from the previous step to determine the neighboring position on the meridian and consequently the correct value of $r^{\prime}$ in the current step.

Fig. 14 shows three milling paths using a particular hyperbolic milling tool and a coverage of the surface by several largest patches. It further demonstrates advantage of general milling tools. In this convex side of the blisk blade, efficient flank milling using traditional conical tools is not possible as a conical tool can have only a single-point (first order) contact with a convex surface. In contrast, using a proper hyperbolic tool and our path planning algorithm, one looks for a higher-order contact and therefore can find a single sweep that covers large portions of the surface and that meets fine machining accuracy (of few micrometers for an object of tens of centimeters). We emphasize again that the best shape of such a tool that, e.g., maximizes the coverage of the input surface goes beyond this paper.

\subsection{Discussion and limitations}

Our method returns patches that meet fine industrial tolerances, however, there are several further challenges that are closely associated to our solutions. The patches are disordered and finding a sequence of patches that, e.g., minimizes the milling time could be very relevant. Moreover, our solution is in the format of a set of patches (an atlas), where many patches have very large overlaps. We used a heurisitic algorithm that takes the largest patch (in terms of surface area) and looks for another patch that has second largest area with small ( $<5 \%$ of area) overlap. Such a searching algorithm is suboptimal; moreover it returns solutions that admit gaps, Fig. 13 middle. Tool-paths with gaps are undesirable from the practical perspective and a more sophisticated algorithms could be applied to extract a subset of patches from our atlas. Alternatively, one can follow the subdivision approach from Ezair and Elber [2018] to segment the whole surface into smaller patches and to simplify the search for globally accessible tool positions.

Another rather unsolved problem are regions close to the boundary of the reference surface. For our experimental purposes, we trimmed one front and one back face of the blade shown in Fig. 11, making the reference geometry open, even though the whole blade is a closed surface. 


\section{Conclusion and Future Work}

We have presented an initialization algorithm that detects initial milling paths of general tools in the context of 5-axis flank CNC machining. The algorithm is a generalization of Bo et al. [2017] for milling tools with generally curved meridians. The algorithm exploits the second order approximation of the point-surface distance and computes a multi-valued vector field that corresponds to 3D directions in which the distance from the surface changes according to the shape of the milling tool. The integration of the multi-valued vector fields gives rise a family of integral curves and, among them, straight line segments are sought after to form a discrete set of positions of the milling axes.

Our algorithm has been tested against benchmark machining datasets as well as synthetic examples. The algorithm recovers the exact solutions and, for a general input that is not an envelope, the computed milling paths approximate the input free-form surface within a fine machining tolerance.

Our research is a step towards custom-shaped manufacturing where a geometrical analysis of an object to be manufactured reveals a set of tools that offer a better approximation quality than the "on-market" milling tools. Even though these custom-shaped tools are object-dependent, their cost is typically negligible compared to the whole manufacturing process, and therefore we believe that the research in this direction is very promising as it not only further improves the machining quality, but also reduces the machining costs.

Acknowledgments. The first author has been partially supported by the National Natural Science Foundation of China (Grant No. 61672187), the Shandong Provincial Key Research \& Development Project (2018GGX103038), and the Open Project Program of the National Laboratory of Pattern Recognition (NLPR) (201800013). The second author has been partially supported by Spanish State Research Agency (Spanish Ministry of Science, Innovation and Universities): BCAM Severo Ochoa excellence accreditation SEV-2017-0718 and by Ramón y Cajal with reference RYC-2017-22649.

\section{References}

Alliez, P., Cohen-Steiner, D., Tong, Y., Desbrun, M., 2007. Voronoi-based variational reconstruction of unoriented point sets, in: Symposium Geometry Processing. Eurographics, pp. 39-48.

Beudaert, X., Lavernhe, S., Tournier, C., 2012. Feedrate interpolation with axis jerk constraints on 5-axis NURBS and $g 1$ tool path. International Journal of Machine Tools and Manufacture 57, 73-82.

Bo, P., Bartoň, M., Plakhotnik, D., Pottmann, H., 2016. Towards efficient 5-axis flank CNC machining of free-form surfaces via fitting envelopes of surfaces of revolution. Computer-Aided Design 79, 1-11.

Bo, P., Bartoň, M., Pottmann, H., 2017. Automatic fitting of conical envelopes to free-form surfaces for flank CNC machining. Computer-Aided Design 91, 84-94.

Calleja, A., Bo, P., González, H., Bartoň, M., López de Lacalle, L.N., 2018. Highly accurate 5-axis flank CNC machining with conical tools. The International Journal of Advanced Manufacturing Technology, 1-11.

Campa, F., de Lacalle, L.L., Lamikiz, A., Sanchez, J., 2007. Selection of cutting conditions for a stable milling of flexible parts with bull-nose end mills. Journal of Materials Processing Technology 191, 279-282.

Chang, J.W., Wang, W., Kim, M.S., 2010. Efficient collision detection using a dual obb-sphere bounding volume hierarchy. Computer-Aided Design 42, 50-57.

Chen, H.Y., Pottmann, H., 1999. Approximation by ruled surfaces. J. Comput. Appl. Math. 102, 143-156.

Chu, C., Huang, W., Hsu, Y., 2008. Machining accuracy improvement in five-axis flank milling of ruled surfaces. International Journal of Machine Tools and Manufacture 48, 914-921.

De Lacalle, L.L., Angulo, C., Lamikiz, A., Sanchez, J., 2006. Experimental and numerical investigation of the effect of spray cutting fluids in high speed milling. Journal of Materials Processing Technology 172, 11-15.

Elber, G., 1994. Accessibility in 5-axis milling environment. Computer-Aided Design 26, 796-802.

Elber, G., Fish, R., 1997. 5-axis freeform surface milling using piecewise rule surface approximation. ASME Journal of Manufacturing Science and Engineering 119, 383-387.

Engin, S., Altintas, Y., 2001. Mechanics and dynamics of general milling cutters.: Part i: helical end mills. International Journal of Machine Tools and Manufacture 41, 2195-2212.

Ezair, B., Elber, G., 2018. Automatic generation of globally assured collision free orientations for 5-axis ball-end tool-paths. Computer-Aided Design 102, 171-181.

Fan, J., Ball, A., 2014. Flat-end cutter orientation on a quadric in five-axis machining. Computer-Aided Design 53, $126-138$.

Farin, G., Hoschek, J., Kim, M., 2002. Handbook of computer aided geometric design. Elsevier.

Flöry, S., Pottmann, H., 2010. Ruled surfaces for rationalization and design in architecture, in: LIFE in:formation. On Responsive Information and Variations in Architecture, pp. 103-109.

Gong, H., Li-Xin, C., Jian, L., 2005. Improved positioning of cylindrical cutter for flank milling ruled surfaces. Computer-Aided Design 37, 1205-1213. 
Gong, H., Wang, N., 2009. Optimize tool paths of flank milling with generic cutters based on approximation using the tool envelope surface. Computer-Aided Design 41, 981-989.

Harik, R.F., Gong, H., Bernard, A., 2013. 5-axis flank milling: A state-of-the-art review. Computer-Aided Design 45, 796-808.

Ikua, B.W., Tanaka, H., Obata, F., Sakamoto, S., 2001. Prediction of cutting forces and machining error in ball end milling of curved surfaces-i theoretical analysis. Precision Engineering 25, 266-273.

Kanungo, T., Mount, D.M., Netanyahu, N., Piatko, C., Silverman, R., Wu, A., 2002. An efficient k-means clustering algorithm: Analysis and implementation. IEEE transactions on pattern analysis and machine intelligence 24, 881-892.

Kim, Y., Bartoň, M., Elber, G., Pottmann, H., 2015. Precise gouging-free tool orientations for 5-axis CNC machining. Computer-Aided Design 58, 220-229.

Larue, A., Altintas, Y., 2005. Simulation of flank milling processes. International Journal of Machine Tools and Manufacture $45,549-559$

Li, C., Bedi, S., Mann, S., 2006. Flank milling of a ruled surface with conical tools - an optimization approach. Int. J. Adv. Manuf. Technol. 29, 1115i-1124.

Li, C., Bedi, S., Mann, S., 2008. Flank millable surface design with conical and barrel tools. Computer- Aided Design and Applications 5, 461-470.

Li, S.X., Jerard, R.B., 1994. 5-axis machining of sculptured surfaces with a flat-end cutter. Computer-Aided Design 26, $165-178$

Lu, Y., Bi, Q., Zhu, L., 2014. Five-axis flank milling of impellers: Optimal geometry of a conical tool considering stiffness and geometric constraints. Proceedings of the Institution of Mechanical Engineers, Part B: Journal of Engineering Manufacture $228,1226-1236$

Machchhar, J., Plakhotnik, D., Elber, G., 2017. Precise algebraic-based swept volumes for arbitrary free-form shaped tools towards multi-axis CNC machining verification. Computer-Aided Design .

Pechard, P.Y., Tournier, C., Lartigue, C., Lugarini, J.P., 2009. Geometrical deviations versus smoothness in 5-axis high-speed flank milling. International Journal of Machine Tools and Manufacture 49, 453-461.

Pottmann, H., Peternell, M., 2000. Envelopes-computational theory and applications, in: Proceedings of Spring Conference on Computer Graphics, pp. 3-23.

Pottmann, H., Schiftner, A., Wallner, J., 2008. Geometry of architectural freeform structures. Int. Math. Nachr. 209, 15-28.

Redon, S., Lin, M.C., Manocha, D., Kim, Y.J., 2005. Fast continuous collision detection for articulated models. Journal of Computing and Information Science in Engineering 5, 126-137.

Redonnet, J., Rubio, W., Dessein, G., 1998. Side milling of ruled surfaces; optimum positioning of the milling cutter and calculation of interference. The International Journal of Advanced Manufacturing Technology 14, $459-465$.

Senatore, J., Landon, Y., Rubio, W., 2008a. Analytical estimation of error in flank milling of ruled surfaces. Computer-Aided Design 40, 595-603.

Senatore, J., Monies, F., Landon, Y., Rubio, W., 2008b. Optimising positioning of the axis of a milling cutter on an offset surface by geometric error minimisation. Int. J. Adv. Manuf. Technol. 37, 861-871.

Senatore, J., Moniès, F., Rubio, W., 2012a. 5-axis flank milling of sculptured surfaces, in: Machining of Complex Sculptured Surfaces. Springer, pp. 33-65.

Senatore, J., Segonds, S., Rubio, W., Dessein, G., 2012b. Correlation between machining direction, cutter geometry and step-over distance in 3-axis milling: Application to milling by zones. Comput. Aided Des. 44, 1151-1160.

Sonthipermpoon, K., Bohez, E., Hasemann, H., Rautenberg, M., 2010. The vibration behavior of impeller blades in the five-axis CNC flank milling process. The International Journal of Advanced Manufacturing Technology 46, 1171-1177.

Sorby, K., Tonnessen, K., Torjusen, J.E., Rasch, F.O., 2000. Improving high speed flank milling operations in multi-axis machines. CIRP Annals-Manufacturing Technology 49, 371-374.

Sprott, K., Ravani, B., 2008. Cylindrical milling of ruled surfaces. Int. J. Adv. Manuf. Technol. 38, 649-656.

Sun, Y., Sun, J., Li, J., Li, W., Feng, B., 2013. Modeling of cutting force under the tool flank wear effect in end milling ti6al4v with solid carbide tool. The International Journal of Advanced Manufacturing Technology 69, 2545-2553.

Várady, T., Martin, R., 2002. Reverse Engineering. In: Handbook of computer aided geometric design. Elsevier.

Wang, C., Elber, G., 2014. Multi-dimensional dynamic programming in ruled surface fitting. Computer-Aided Design 51, 39-49.

Wang, X.C., Ghosh, S.K., Li, Y.B., Wu, X.T., 1993a. Curvature catering - a new approach in manufacture of sculptured surfaces (part 1. theorem). Journal of Materials Processing Technology 38, 159-175.

Wang, X.C., Ghosh, S.K., Li, Y.B., Wu, X.T., 1993b. Curvature catering - a new approach in manufacture of sculptured surfaces (part 2. methodology). Journal of Materials Processing Technology 38, 177-193.

Warkentin, A., Ismail, F., S., B., 2000. Comparison between multi-point and other 5-axis tool positioning strategies. Journal of Machine Tools \& Manufacture 40, 185-208.

Xu, K., Wang, J., Chu, C.H., Tang, K., 2017. Cutting force and machine kinematics constrained cutter location planning for five-axis flank milling of ruled surfaces. Journal of Computational Design and Engineering 4, $203-217$.

Zhang, X., Zhang, J., Pang, B., Zhao, W., 2016. An accurate prediction method of cutting forces in 5-axis flank milling of sculptured surface. International Journal of Machine Tools and Manufacture 104, 26-36.

Zhao, H., Zhang, H., Xin, S., Deng, Y., Tu, C., Wang, W., Cohen-Or, D., Chen, B., 2018. Dscarver: decompose-and-spiral-carve for subtractive manufacturing. ACM Transactions on Graphics (TOG) 37, 137.

Zhu, L., Lu, Y., 2015. Geometric conditions for tangent continuity of swept tool envelopes with application to multi-pass flank milling. Computer-Aided Design 59, 43-49.

Zhu, L., Zheng, G., Ding, H., Xiong, Y., 2010. Global optimization of tool path for five-axis flank milling with a conical cutter. Computer-Aided Design 42, 903-910. 